\title{
Bom para alguns, ótimo para outros
}

O sildenafil, um inibidor das isoenzimas fosfodiesterases (PDE) 5 e 6 , desenvolvido originalmente para o tratamento da disfunção erétil,(1) tem-se mostrado bastante eficaz como vasodilatador pulmonar. 0 sildenafil inibe a hidrólise e aumenta a concentração da guanosina monofosfato cíclico (GMPc) na célula muscular lisa da parede vascular (CML), o segundo mensageiro para a ação intracelular do óxido nítrico (ON).

$O$ acúmulo do GMPc inibe a ação da PDE-3, resultando em redução da hidrólise e conseqüente aumento da concentração da adenosina monofosfato cíclico (AMPc), o segundo mensageiro dentro das CML para as prostaciclinas. ${ }^{(2,3)}$ Então, o sildenafil exerce ação abrangente dentro da CML, o que foi verificado em alguns trabalhos experimentais. ${ }^{(4,5)}$ O s tecidos com maior concentração de PDE-5 são os corpos cavernosos, O CML pulmonar e a retina. (2)

Os registros sobre o emprego clínico do sildenafil no tratamento da hipertensão arterial pulmonar (HAP) iniciaram com relatos de caso. ${ }^{(6)}$ Seguiram-se com seu emprego em séries de casos como fármaco associado aos prostanóides ou ao óxido nítrico, com o objetivo de aumentar e prolongar as ações dos fármacos principais. ${ }^{(7-14)}$ Seguem aspectos mais relevantes de alguns artigos originais.

Os referidos estudos permitem considerar que: (1) o sildenafil é um vasodilatador eficiente, com potência superior ao on e comparável às doses plenas de prostanóides parenterais; (2) seus efeitos se exercem, notadamente, sobre a circulação pulmonar, sobre os parâmetros hemodinâmicos e sobre o intercâmbio de gases; (3) o sildenafil atua sinergicamente com os vasodilatadores com os quais foi até agora testado, como o ON e os prostanóides, mesmo em pequenas doses; (4) tanto em relatos isolados como em série de casos, o sildenafil tem confirmado efeitos favoráveis e poucos efeitos adversos; (5) o chamado efeito antimitogênico (antiproliferativo ou de reversão do remodelamento), atribuído a outros fármacos vasodilatadores, ainda não foi confirmado com o sildenafil, cuja ação vasodilatadora é atribuída a um potente efeito amplificador dos mecanismos vasorregulatórios na circulação pulmonar; (6) ainda se está carente de um estudo com delineamento de ensaio clínico de padrão A para avaliar seus efeitos de longo prazo e seus efeitos adversos; (7) desde o trabalho original,* tem havido evidências de efeitos adversos do sildenafil sobre a visão, por lesão na retina (um estudo experimental em camundongos produziu retinite pigmentosa dose-dependente); ${ }^{(15)}$ os efeitos adversos relacionados encontrados até agora têm sido leves, com náuseas, cefaléia e hipotensão dosedependentes; (8) o sildenafil pode ser uma nova opção para o tratamento isolado ou combinado da hipertensão pulmonar primária (HPP), HAP associada a doenças colágeno-vasculares e mesmo uma alternativa de tratamento clínico para a hipertensão pulmonar tromboembólica crônica (HPTEC) inoperável.

No presente número do J Pneumol são apresentados dois relatos de casos com emprego de sildenafil, ${ }^{(16,17)}$ com significativa contribuição.

Souza et al. ${ }^{16}$ usaram sildenafil em uma paciente de 33 anos de idade com lúpus eritematoso sistêmico e síndrome antifosfolipídeo, com elevação progressiva da pressão sistólica de artéria pulmonar progressiva (PSAP), que passou de $74 \mathrm{mmHg}$ a $102 \mathrm{mmH} \mathrm{g}$, em 18 meses de observação, sob tratamento convencional com diltiazem, warfarin e pulsos de ciclofosfamina e metilprednisolona. Sildenafil foi introduzido de forma cautelosa e lentamente progressiva, na dose de $25 \mathrm{mg}$ ao dia e elevada até $100 \mathrm{mg}$ ao dia, divididas em duas tomadas diárias. Em seis meses de tratamento a PSAP reduziu-se para $67 \%$ (queda de $34 \%$ ), sem efeitos adversos. A justificada estratégia cautelosa de uso do sildenafil, associada à boa resposta pressórica, sugerem que talvez não tenha sido atingido o potencial de resposta.

Silva et al. ${ }^{(17)}$ apresentaram um caso de hipertensão pulmonar primária (HPP) grave - PSAP $116 \mathrm{mmHg}$ - em jovem de 21 anos de idade com piora súbita da saturação arterial de oxigênio (de 95\% para 65\% em ar ambiente) após instalação de shunt direita-esquerda por abertura do forame oval (não identificada em exame anterior). Foi iniciado sildenafil na dose de $25 \mathrm{mg}$ a cada oito horas, com rápidos aumentos diários de $25 \mathrm{mg}$ por dose até atingir-se $225 \mathrm{mg} /$ dia (provavelmente $75 \mathrm{mg}$ a cada oito horas). Houve melhora progressiva no quadro clínico e na saturação arterial, tendo havido reversão do shunt em cerca de 30 dias de tratamento. Fato marcante foi a ma-

\footnotetext{
* No último congresso da A merican Thoracic Society-ATS realizado em Seatle em maio de 2003, o Dr. LJ Rubin, ao apresentar o sildenafil, disse que seus efeitos são bons para alguns e ótimos para outros. Referia-se implicitamente à opinião dos pesquisadores, mas a platéia achou graça.
} 
nutenção da PSAP em valores inalterados $(109 \mathrm{mmH}$ ), não obstante a interrupção do shunt D-E, fato atribuído pelos autores como decorrente do aumento global da capacitância da circulação pulmonar, permitindo o alívio do volume sistólico final do ventrículo direito, com conseqüente melhora do débito cardíaco. Lamenta-se a falta de medidas hemodinâmicas que avaliassem os demais parâmetros pertinentes, que, provavelmente, corroborariam a interpretação dos autores.

Assim, o sildenafil está entre nós.

\section{Sérgio Saldanha Menna Barreto (te SBPt) Professor Titular da Faculdade de Medicina, Universidade Federal do Rio Grande do Sul. Título de especialista pela Sociedade Brasileira de Pneumologia e Tisiologia.}

\section{REFERÊNCIAS}

1. Goldstein I, Lue TF, Padma-Nathan H, Rosen RC, Steers WD, Wicker PA. O ral sildenafil in the treatment of erectile function. Sildenafil Study Group. N Engl J Med 1998;338:1397-404.

2. Beavo JA. Cyclic nucleotide phosphodiesterases: functional implications of multiple isoforms. Physiol Rev 1995;75:725-48.

3. Corbin J D, Francis SH. Cyclic GMP phosphodiesterase-5: a target of sildenafil. J Biol Chem 1999;274:13729-32.

4. Ichinose F, Erana-Garcia J , H romi J, Raveh Y, J ones R, Krim L, et al. Nebulized sildenafil is a selective pulmonary vasodilator in lambs with acute pulmonary hypertension. Crit Care Med 2001;29:1000-5.

5. Kleinsasser $A$, Loeckinger A, Hoermann C, Puehringer F, Mutz N, Bartsch G, Lindner KH. Sildenafil modulates hemodynamics and pulmonary gas exchange. Am J Respir Crit Care Med 2001;163:339-43.

6. Prasad S, Wilkinson J, Gatzoulis MA. Sildenafil in primary pulmonary hypertension. N Engl J Med 2000;343:1342.
7. Wilkens H, Guth A, König J , Forestier N, Cremers B, Hennen B, et al. Effect of inhaled iloprost plus oral sildenafil in patients with primary pulmonary hypertension. Circulation 2001;104:1218-22.

8. Ghofrani HA, Wiedermann R, Rose F, Olschewski H, Schermuly RT, Weissman $\mathrm{N}$. et al. Combination therapy with oral sildenafil and inhaled iloprost for severe pulmonary hypertension. Ann Intern Med 2002; 136:515-22.

9. Ghofrani HA, Rose F, Schermuly RT et al.Oral sildenafil as long-term adjunct therapy to inhaled iloprost in severe pulmonary arterial hypertension. J Am Coll Cardiol 2003;42:158-64.

10. Lepore J J, Maroo A, Pereira NL, et al. Effect of sildenafil on the acute pulmonary vasodilator response to inhaled nitric oxide in adults with primary pulmonary hypertension. Am J Cardiol 2002;90:677-80.

11. Ghofrani HA, Wiedermann R, Rose F, et al. Sildenafil for treatment of lung fibrosis and pulmonary hypertension: a randomised controlled trial. Lancet 2002;360:895-900.

12. Stiebellehner $L$, Petkov $V$, Vonbank $K$, et al. Long-term treatment with oral sildenafil in addition to continuous IV epoprostenol in patients with pulmonary arterial hypertension. Chest 2003;123:1293-5.

13. Ghofrani HA, Schermuly RT, Rose F, et al. Sildenafil for long term treatment of nonoperable chronic thromboembolic pulmonary hypertension. Am J Respir Crit Care Med 2003;167:1139-41.

14. Sastry BK, Narasimhan C, Reddy NK et al. A study of clinical efficacy of sildenafil in patients with primary pulmonary hypertension. Indian Heart J 200;54:410-5.

15. Behn D, Potter MJ . Sildenafil-mediated reduction in retinal function in heterozygous mice lacking the gamma-subunit of phosphodiesterase. Invest O phthalmol Vis Sci 2001;42:523-7.

16. Souza EJ R, Garib J R, Garib NM, Pádua PM. Sildenafil no tratamento da hipertensão pulmonar associada ao LES e síndrome antifosfolipídio. J Pneumol 2003;29:302-4.

17. Silva SM, Valeri CB, Bogossian HB, J ardim C, Demarzos SE, Souza $R$. Resolução do shunt direita-esquerda após o uso de sildenafil como tratamento da hipertensão pulmonar primária. J Pneumol 2003;29: 305-8. 